\title{
Interleukin-33 Predicts Poor Prognosis and Promotes Renal Cell Carcinoma Cell Growth Through its Receptor ST2 and the JNK Signaling Pathway
}

\author{
Chang-wen Wu $\mathrm{W}^{\mathrm{a}} \quad$ Yi-guo Wu $\mathrm{Wu}^{\mathrm{a}}$ Cheng Cheng ${ }^{\mathrm{a}}$ Zheng-dong Hong ${ }^{\mathrm{a}}$ Zi-min Shi ${ }^{\mathrm{a}}$ \\ Shuang-quan Lin ${ }^{a}$ Jie Lib Xiao-yi He ${ }^{c}$ An-yi Zhu \\ aDepartment of Urology Surgery, Second Affiliated Hospital of Nanchang University, Nanchang, \\ Jiangxi Province, bjiangxi Province Key Laboratory of Molecular Medicine, Nanchang, Jiangxi Province, \\ Institute of Translational Medicine, Nanchang University, Nanchang, Jiangxi Province, PR China
}

\section{Key Words}

Renal cell carcinoma $\bullet \mathrm{IL}-33 \cdot \mathrm{ST} 2 \cdot \mathrm{JNK} \cdot$ Prognosis

\begin{abstract}
Background/Aims: Renal cell carcinoma (RCC) is currently the ninth most common cancer in men. Interleukin (IL)-33 expression has previously been associated with a number of cancers; however, its biological role in RCC is poorly understood. In this study, we sought to elucidate the role of IL-33 in RCC. Methods: Serum IL-33 levels were measured by ELISA. IL-33 expression in clinical RCC samples was examined by immunocytochemistry. The proliferation and apoptosis rate of RCC were determined by CCK8 and flow cytometry. Mcl1 and Bcl-2 expression were measured by quantitative real-time PCR and western blotting. JNK expression were measured by western blotting and flow cytometry. The in vivo role of IL-33 in RCC tumorigenesis was examined by animal models. Results: We found that increased expression of IL-33 in RCC was associated with tumor-lymph node-metastasis (TNM) stage and inversely correlated with prognosis. IL-33 enhances RCC cell growth in vivo and stimulates RCC cell proliferation and prevents chemotherapy-induced tumor apoptosis in vitro. Furthermore, we demonstrated that IL-33 promotes RCC cell proliferation and chemotherapy resistance via its receptor ST2 and the JNK signaling activation in tumor cells. Conclusion: Our findings suggest that targeting IL-33/ST2 and JNK signaling may have potential value in the treatment of RCC.

(C) 2018 The Author(s)

Published by S. Karger AG, Basel
\end{abstract}

\section{Introduction}

Renal cell carcinoma (RCC) is the ninth most commonly diagnosed cancer in men worldwide and causes approximately 100, 000 deaths each year [1]. Nephrectomy is the C.-w. Wu and Y.-g. Wu contributed equally to this work. 


\section{Cellular Physiology Cell Physiol Biochem 2018;47:191-200

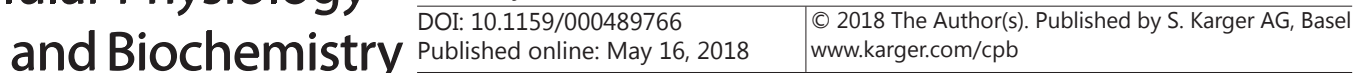 \\ Wu et al.: IL-33 Promotes RCC Cell Proliferation}

standard surgical therapy for primary localized RCC[2]. However, in patients with advancedstage RCC, targeted therapy is the treatment of choice because of the cancer's intrinsic resistance to conventional chemotherapy and radiotherapy. Currently, there are several targeted drugs for RCC, including tyrosine kinase inhibitors, antibodies against vascular endothelial growth factor, and mTOR pathway inhibitors $[3,4]$. However, these drugs do not improve overall survival and progression-free survival in all cases. Therefore, the development of novel drugs is important.

Interleukin (IL)-33, which is expressed by non-hematopoietic cells [5, 6], is a relatively new identified member of the IL-1 family of cytokines. IL-33 exerts its biological functions through binding and activation of its receptor ST2, a member of the Toll-like receptor superfamily $[5,6]$. Previous studies have demonstrated that IL-33 is usually localized in the cell nucleus as an alarmin that signals to local immune cells in different murine model systems [6-14]. Interestingly, it has been reported that IL-33 can protect against infectioninduced tissue damage [13] and also promote biliary repair [15]. Thus, IL-33 has a variety of biological activities in different pathological models. Thus, the role of IL-33 in tumors is under debate. IL-33 can promote antitumor CD8+ T cell responses $[7,16]$ and induce programmed oncosis of the ST2L-positive low-metastatic cells [17] in experimental mouse tumor models. However, IL-33 is associated with cancer progression in several cancer models [17-19]. Nonetheless, the potential biological effect of IL-33 on RCC is poorly understood.

In this study, we investigated the role of IL-33 in RCC. We have found that increased expression of IL-33 in RCC is associated with tumor-lymph node-metastasis (TNM) stage and inversely correlated with prognosis. IL-33 enhances RCC cell growth in vivo and stimulates RCC cell proliferation and prevents chemotherapy-induced tumor apoptosis in vitro. Furthermore, we have demonstrated that IL-33 promotes RCC cell proliferation and chemotherapy resistance via its receptor ST2 and the JNK signaling activation in tumor cells. Our work suggests that targeting IL-33/ST2 and JNK signaling may be potentially applicable in treating patients with RCC.

\section{Materials and Methods}

\section{Clinical tissue specimens}

A total of 46 patients ( 24 men and 22 women) with pathologically confirmed RCC were enrolled (Second Affiliated Hospital of Nanchang University, Nanchang, China). All patients had undergone radical nephrectomy between 2009 and 2012. The patients were classified according to World Health Organization criteria and staged according to the TNM classification system. The pathologic type for all patients was clear cell RCC. Samples were obtained from the patients after obtaining signed informed consent in the Second Affiliated Hospital of Nanchang University, Nanchang, China.

\section{Cell culture}

Renal cancer cell lines (7860 and OSRC2) were purchased from the American Tissue Culture Collection (Rockville, MD, USA). The 7860 and OSRC2 cells were grown in RPMI1640 medium (Hyclone, Logan, UT, USA) supplemented with 10\% fetal bovine serum (Hyclone, 10270) and 1\% penicillin/streptomycin (Invitrogen, Carlsbad, CA, USA; 15140).

\section{RCC cell proliferation assay}

RCC cells were plated in six well plates (Corning, USA) in RPMI1640 medium (Lonza, Switzerland) at a density of 1, 000-5, 000 viable cells/well. RCC cells were treated with recombinant IL-33 (Peprotech, USA) at different doses. The JNK inhibitor, SP600125 (Cayman, USA), or anti-human ST2 antibody (Clone \# 97203, R\&D systems, USA) were added. The proliferation was measured by CCK8.

RCC cell apoptosis assay

RCC cells were plated in six well plates (Corning) in RPMI1640 medium (Lonza) at a density of 1, 000-5, 000 viable cells/well in the presence of 5-Fu at different doses. RCC cells were treated with $50 \mathrm{ng} /$ 


\section{Cellular Physiology Cell Physiol Biochem 2018;47:191-200 \begin{tabular}{l|l} 
and Biochemistry Published online: May 16, 2018 & $\begin{array}{l}\text { D) } 2018 \text { The Author(s). Published by S. Karger AG, Basel } \\
\text { www.karger.com/cpb }\end{array}$ \\
\hline
\end{tabular} \\ Wu et al.: IL-33 Promotes RCC Cell Proliferation}

mL recombinant IL-33 (Peprotech). The JNK inhibitor, SP600125 (10 $\mu \mathrm{g} / \mathrm{mL}$, Cayman, USA), or anti-human ST2 antibody ( $1 \mu \mathrm{g} / \mathrm{mL}$, R\&D systems) were added. The apoptosis rate was measured by flow cytometry.

\section{SiRNA Transfection}

ST2 expression was knockdown using a synthetic siRNA sequence against ST2. RCC cells were transfected with human ST2 siRNAs or control siRNA using Lipofectamine RNAi MAX (Life Technologies) according to manufacturer's protocols.

\section{Immunohistochemical analysis}

Formalin-fixed, paraffin-embedded RCC tissue sections $(5 \mathrm{~mm})$ were dewaxed and rehydrated before an antigen retrieval step. Sections were then incubated with anti-human primary antibodies for IL-33 (Enzo Life Sciences) or matching IgG isotypes overnight. Slides were then stained with species-specific biotinylated secondary antibodies (R\&D Systems), streptavidin horseradish peroxidase (HRP), and detected with substrate AEC (3-amino-9-ethylcarbazole; Vector Laboratories). Subsequently, slides were counterstained with hematoxylin. IL-33 expression was evaluated according to the intensity and extent of staining. The proportion of stained cells per specimen was semi-quantitatively evaluated and scored as follows: 0 for staining $\leq 1 \%$; 1 for $2-25 \%$; 2 for $26-50 \%$; 3 for $51-75 \%$; and 4 for $>75 \%$ of the examined cells. The staining intensity was stratified as follows: 0 , negative staining; 1 , weak staining; 2 , moderate staining; and 3 , strong staining. The results were evaluated using the following formula: total score $=$ proportion score $\times$ intensity score. A total score of $0-12$ was graded as negative (-; score: $0-4)$ or positive (+; score: $5-12$ ).

\section{Western blotting}

Equal amounts of protein $(45 \mu \mathrm{g})$ from each sample were separated by 10\% SDS-PAGE and transferred to PVDF membranes (Millipore). The proteins were then incubated overnight at $4^{\circ} \mathrm{C}$ with primary antibodies against human phospho-JNK, JNK, phospho-ERK, ERK, phospho-P38, and P38, (Cell Signaling Technology). Following a $30 \mathrm{~min}$ wash, the membranes were incubated with an HRP-conjugated secondary antibody for $1 \mathrm{~h}$ at room temperature. After an additional wash for $30 \mathrm{~min}$, the membranes were visualized by enhanced chemiluminescence (Millipore) and recorded on Kodak film.

\section{Flow cytometry}

The Abs used for flow cytometry were FITC-, APC -, or PE -conjugated mouse anti-human ST2, P-P38, P-ERK, and P-JNK Abs from BD. The cultured cells were collected, washed twice, and resuspended in $100 \mu \mathrm{l}$ of PBS containing $0.1 \%$ BSA. These cells were stained and labeled with either ST2 Abs or the appropriate isotype controls. The cells were incubated on ice for $30 \mathrm{~min}$, washed with PBS containing $0.1 \% \mathrm{NaN} 3$ and $0.5 \% \mathrm{BSA}$, and then fixed in $1 \%$ paraformaldehyde solution. For the intracellular staining of cultured cells, the cells were fixed and permeabilized with fixation/permeabilization solution (eBioSciences, San Diego, CA) for $15 \mathrm{~min}$ at room temperature. Then, the cells were washed again and stained with monoclonal antibodies against P-JNK, P-P38, and P-ERK for $20 \mathrm{~min}$ at room temperature. Analyses were performed using FACScan and CellQuest software (BD Biosciences).

\section{Analysis of RCC cell apoptosis by Flow cytometry}

RCC cell apoptosis was quantified using a PI/Annexin V apoptosis detection kit according to the manufacturer's instructions (Invitrogen). The binding of Annexin V-FITC and PI to the cells was measured by FACS Calibur (BD Biosciences, NJ, USA) using Cell Quest software.

Table 1. Primer sequences of target genes

\begin{tabular}{|c|c|c|c|}
\hline Genes & Primer sequence $(50-30)$ & Amplicon size (bp) & Annealing temp $\left({ }^{\circ} \mathrm{C}\right)$ \\
\hline Mcl-1 For: & ACGGCCTTCCAAGGCAT & 103 & 64 \\
\hline Rev: & TTGTTACGCCGTCGCTGA & & \\
\hline$\beta$-actin For: & CACGAAACTACСTTCAАСТCC & 265 & 54 \\
\hline Rev: & CATACTCCTGCTTGCTGATC & & \\
\hline Bcl2 For: & AACGATACTAAATGCAA & 125 & 58 \\
\hline Rev: & TACACGCATACTACCTG & & \\
\hline
\end{tabular}




\section{Cellular Physiology Cell Physiol Biochem 2018;47:191-200

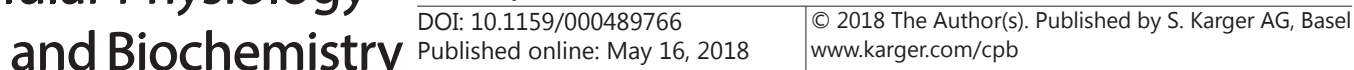 \\ Wu et al.: IL-33 Promotes RCC Cell Proliferation}

\section{Quantitative real-time PCR}

Total RNA was extracted from RCC cells using Trizol reagent. The cDNAs were synthesized using a reverse transcription kit according to the manufacturer's instruction. Quantitative RT-PCR was performed using human-specific primers for the quantification of Mcl-1 (Table 1). $\beta$-actin was used as an internal control. Reactions were performed using SYBR-Green PCR mix (Applied Biosystems, Shanghai, China) in the Bio-Rad CFX96 Real-Time System.

\section{Animal Models}

Six to eight-week-old male nude BALB/c mice (Beijing HFK Bioscience Co., Ltd, China) were used in the RCC experiments. All experiments were conducted according to the Guidelines for the Care and Use of Laboratory Animals and approved by the Ethics Committee of Second Affiliated Hospital of Nanchang University, Nanchang, China. The cells (106) were subcutaneously injected into nude mice. Neutralizing antibody against either IL-33 (Clone \#40015D) or ST2(200ug/mice) was injected every two days for two weeks after RCC cell injection. Tumor size was measured three times weekly with a Vernier caliper. Tumor volume was calculated based on three perpendicular measurements.

\section{Statistical analysis}

The results are expressed as the means \pm SEM. The statistical significance of differences between groups was determined by the Student's t-test. SPSS statistical software (version 13.0) was used for all statistical analyses. All data were analyzed using two-tailed tests unless otherwise specified, and $\mathrm{P}<0.05$ was considered statistically significant. ${ }^{*} \mathrm{P} \leq 0.05,{ }^{* *} \mathrm{P} \leq 0.01,{ }^{* * *} \mathrm{P} \leq 0.001$.

\section{Results}

\section{Increased expression of IL-33 correlates with RCC patient prognosis}

Serum samples were collected from 10 patients with RCC and 10 controls. Serum IL-33 levels were measured by ELISA. IL-33 levels in the serum of RCC patients were significantly elevated in comparison with that of healthy volunteers $(\mathrm{P}<0.01$, Fig. $1 \mathrm{~A})$.

IL-33 expression in clinical RCC samples was examined by immunocytochemistry (Fig. 1B). Forty-six patients were included and classified into two groups: high IL-33 group (IL-33 expression fold changes $\geq$ median fold changes, $\mathrm{n}=$ 29) and low IL-33 group (IL-33 expression fold changes $<$ median fold changes, $n=17$ ). Clinicopathological factors were compared between the two groups (Table 1). Elevated expression of IL-33 in clinical samples was correlated with $\mathrm{T}$ stage $(\mathrm{P}$ $<0.05$, Table 1) and lymph node metastasis $(\mathrm{P}<0.05$, Fig. $1 \mathrm{C})$. However, IL-33 overexpression was not associated with patient age, gender, or location. Kaplan-Meier survival curves showed that patients with high expression levels of IL-33 (n $=29$ ) had shorter overall survival than those with low expression levels of IL-33 ( $\mathrm{n}=17, \mathrm{P}=0.0073$, log rank test; Fig. 1C). These results

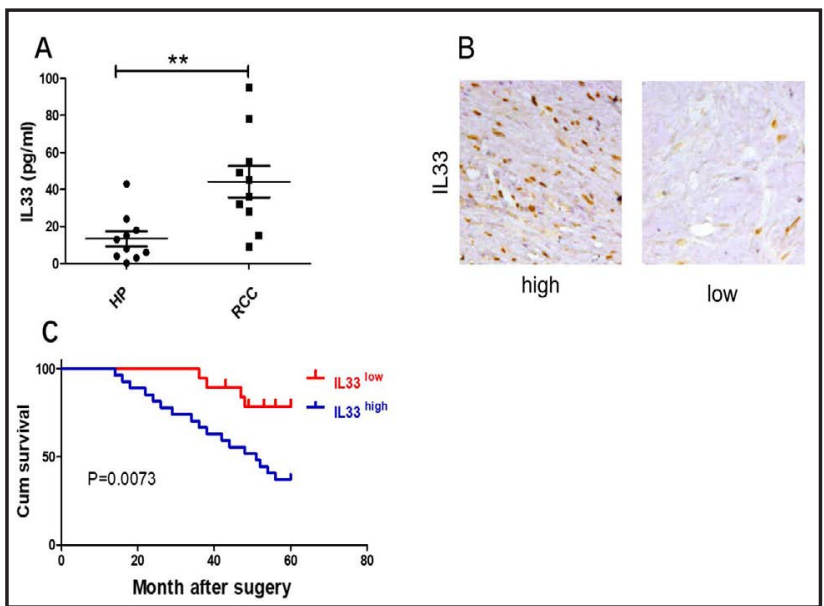

Fig. 1. Increased expression of IL-33 correlates with RCC patient prognosis(A) Elevated expression of IL-33 was detected in RCC clinical samples by ELISA. (B) IL-33 expression was detected with conventional immunohistochemical staining in the human RCC tissues. The representative images of IL-33 high and low expression in RCC tissues are shown. (C) Patients with high expression of IL-33 had a short overall survival after surgery. (D) IL-33 expression and clinicopathological characteristics of RCC. 
indicated that IL-33 may be an independent prognostic factor in RCC and may promote RCC progression and development.

IL-33 promotes RCC
cell proliferation
and prevents
che motherapy-
induced tumor
apoptosis
To test the effects of IL-33 on RCC cell proliferation and 5-fluorouracil (5-Fu) treatment efficacy, we examined the proliferation and apoptosis rate by CCK8 and flow cytometry. We found that IL-33 induced the proliferation of 7860 cells (Fig. 2A) and OSRC2 cells (Fig. 2B) in a dose-dependent manner. Furthermore, IL-33 reduced 7860 cell (Fig. 2C) and OSRC2 cell (Fig. 2D) apoptosis induced by 5 -Fu treatment. In addition, IL-33 increased the Mcl1 expression in 7860 cells

(Fig. 2E and I) and OSRC2 cells (Fig. 2F and I) but decreased Bcl-2 expression in 7860 cell (Fig. 2G and I) and OSRC2 cells (Fig. 2H and I) at the protein and mRNA levels. These findings indicate that IL-33 promotes RCC cell proliferation and prevents chemotherapy-induced tumor apoptosis.

\section{ST2 is crucial for IL-33-mediated RCC cell proliferation and chemotherapy resistance}

To further confirm the role of IL-33 in RCC biology, we detected ST2 expression by flow cytometry in two RCC cell lines (Fig. 3A). Neutralizing monoclonal antibody against ST2 (anti-ST2 mAb) blocked the effects of IL-33 on RCC cell proliferation (Fig. 3B-C) and apoptosis induced by 5-Fu treatment (Fig. 3D-E). Furthermore, we designed a knockdown ST2 siRNA sequence and confirmed the knockdown efficiency of siRNA by flow cytometry (Fig. 3F). As shown in Fig. 3G-J, knockdown of ST2 reduced the promotional effect of IL-33 on the proliferation and drug resistance of RCC.

IL-33 promotes RCC cell proliferation and chemotherapy resistance via JNK activation. Next, we explored the molecular mechanisms by which IL-33 promotes RCC cell proliferation and chemotherapy resistance. It has been reported that IL-33 binds ST2 and activates ERK, c-Jun N-terminal kinase (JNK), and p38 mitogen-activated protein kinase (MAPK) in murine cells [19]. We examined P-P38, P-ERK, and P-JNK expression in RCC cells in the presence or absence of IL-33 by western blotting. The western blotting results showed that IL-33 induced 


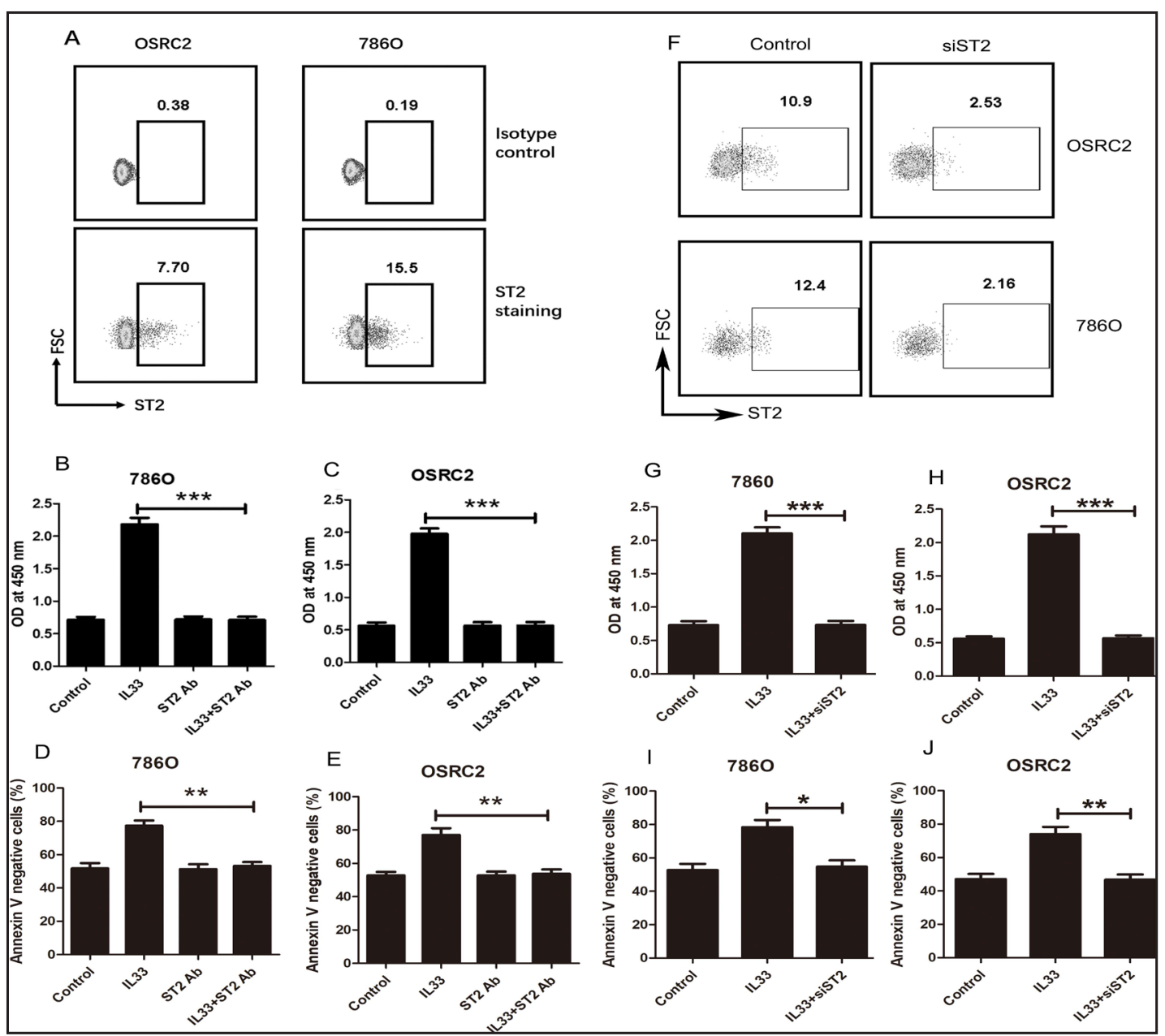

Fig. 3. IL-33 promotes RCC cell proliferation and chemotherapy resistance via its receptor ST2(A) Expression of ST2 protein in RCC cells. OSRC2 and 7860 cells were stained with specific rabbit anti-human ST2 $\mathrm{Ab}$ and FITC-conjugated goat anti-rabbit IgG. The expression of ST2 was determined by flow cytometer analyzer and expressed as the percentage of ST2+ cells in total RCC cells. One of 3 experiments is shown. (B-C) Effects of anti-ST2 on the role of IL-33-mediated RCC cell proliferation. RCC cells were cultured with or without IL-33 (50 ng/mL) for 48 hours and anti-ST2 antibody $(1 \mu \mathrm{g} / \mathrm{mL})$ was added to the culture. The cell proliferation was determined using the CCK-8 assay. Results are expressed as the mean numbers of proliferation \pm SEM. Each column represents three independent experiments. ${ }^{* * *} \mathrm{P}<0.001$. (D-E) Effects of anti-ST2 on the role of IL-33-mediated RCC chemotherapy. RCC cells were cultured with or without IL-33 $(50 \mathrm{ng} / \mathrm{mL})$ for 48 hours and anti-ST2 antibody $(1 \mu \mathrm{g} / \mathrm{mL})$ was added to the culture, and then RCC cells were subsequently exposed to 5 -Fu for 24 hours. The cell viability was determined by flow cytometry. Results are expressed as the mean \pm SEM. ${ }^{* *} \mathrm{P}<0.01$. (F) Flow cytometry of RCC cells transfected with either ST2-siRNA (siST2) or Control-siRNA (Control). (G-H) Effects of siST2 on the role of IL-33-mediated RCC cell proliferation. Results are expressed as the mean numbers of proliferation \pm SEM. Each column represents three independent experiments. ${ }^{* * *} \mathrm{P}<0.001$. (I-J) Effects of siST2 on the role of IL-33-mediated RCC chemotherapy. Results are expressed as the mean \pm SEM. ${ }^{* *} \mathrm{P}<0.01$.

JNK phosphorylation 30 min after addition and further increased JNK phosphorylation at 60 min, but was unable to induce phosphorylation of P38 and ERK (Fig. 4A). Moreover, we examined the effect of IL-33 on P38, ERK, and JNK phosphorylation at 30 min by flow cytometry. As shown in Fig. 4A-B, IL-33 expression resulted in increased P-JNK expression, but did not cause P38 and ERK phosphorylation (Fig. 4B-C). Thus, we speculate that the JNK signaling pathway is involved in IL-33-mediated renal cell proliferation and chemoresistance. 
As shown in Fig. 4D, treatment with a JNKspecific inhibitor, SP600125, abolished IL-33-mediated JNK phosphorylation, as well as the effects of IL-33 on RCC cell proliferation (Fig. $4 \mathrm{E}-\mathrm{F})$ and chemotherapy resistance (Fig. 4G-H).

\section{IL-33 promotes RCC} cancer growth via its receptor ST2 and JNK activation in vivo

To examine the in vivo role of IL-33 in RCC tumorigenesis, we inoculated 7860 and OSRC2 RCC cells into nude mice. We observed that the growth of IL-33treating 7860 and OSRC2 tumors was greater (Fig. $5 \mathrm{~A}-\mathrm{B})$ than that of normal controls. Both ST2 Ab and SP600125 could abolish the effects of IL-33 on 7860 and OSRC2 tumor growth in vivo (Fig. 5A-B). Moreover, we observed that knockdown of ST2 reduced 7860 and OSRC2 tumor growth (Fig. 5C-D) compared to control siRNA, and treatment with a neutralizing antibody against either IL-33 or ST2 could reduce 7860 and OSRC2 tumor growth in vivo (Fig. 5C-D).

\section{Discussion}

In this study, we demonstrate that increased expression of IL-33 in RCC was associated with advanced TNM stage and inversely correlated with prognosis. IL-33 promoted RCC cell proliferation and prevented 5-Fu-induced tumor cell apoptosis through ST2 and the JNK signaling pathway.

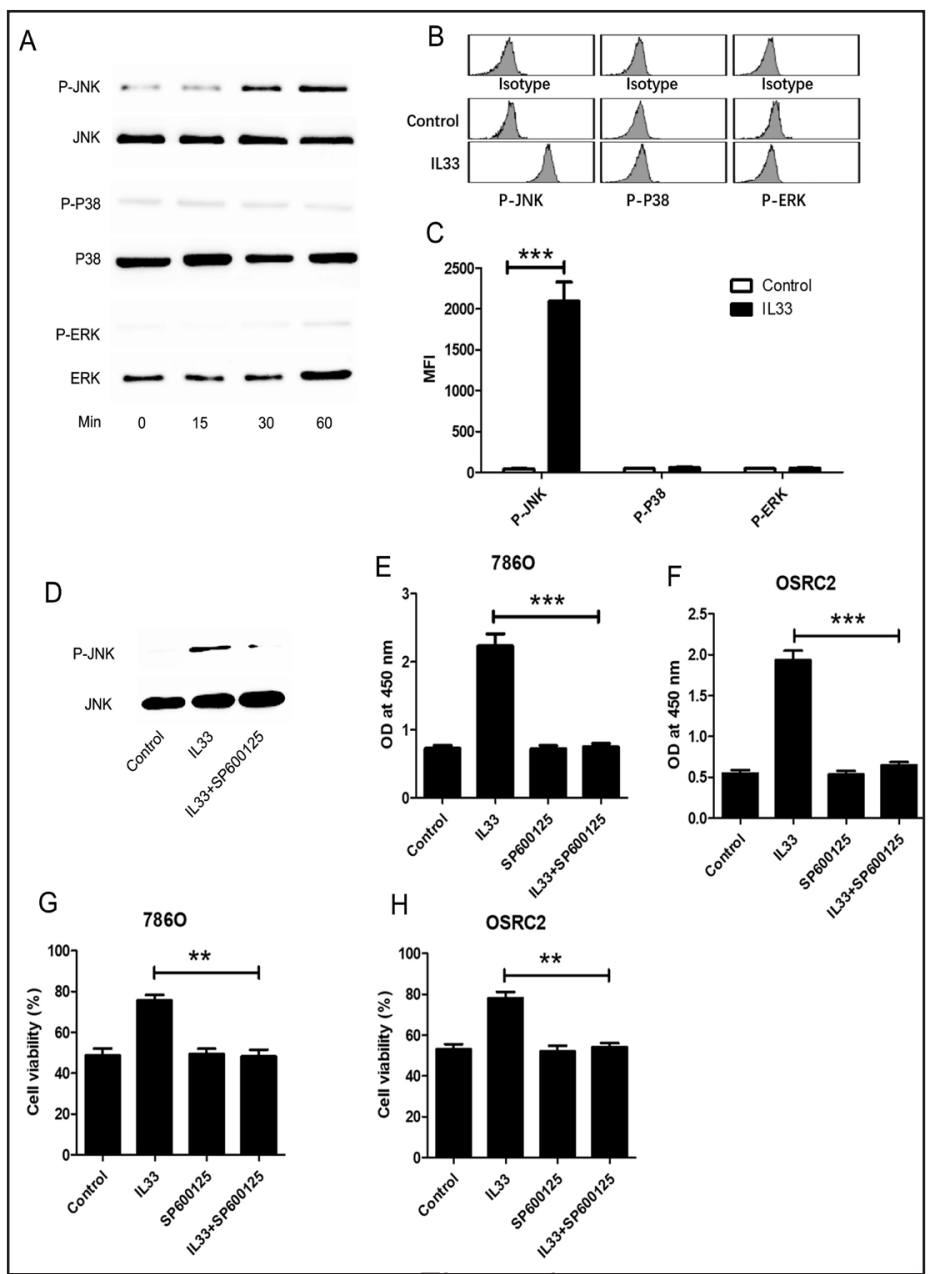

Fig. 4. IL-33 promotes RCC cell proliferation and chemotherapy resistance via JNK activation(A) 7860 cells were stimulated with IL-33 $(50 \mathrm{ng} / \mathrm{mL}$ ) for different times and then harvested for western blotting detection of P-JNK, P-P38, P-ERK. One of 3 experiments is shown. (B-C) 7860 cells were stimulated with IL-33 (50 ng/mL) for $30 \mathrm{~min}$ and then harvested for flow cytometry detection of P-JNK, P-P38, PERK. (B) One of 3 experiments is shown. (C) Each column represents three independent experiments. ${ }^{* * *} \mathrm{P}<0.001$. (D) Effects of the JNK inhibitor SP600125 on IL-33-mediated JNK phosphorylation. One of 3 experiments is shown. (E-F) Effects of the JNK inhibitor SP600125 on RCC cell proliferation. RCC cells were cultured with or without IL-33 (50 ng/mL) for 48 hours and SP600125 $(10 \mu \mathrm{g} / \mathrm{mL})$ was added to the culture. The cell proliferation was determined using the CCK-8 assay. Results are expressed as the mean numbers of proliferation \pm SEM. Each column represents three independent experiments. ${ }^{* * *} \mathrm{P}<0.001$. (G-H) Effects of the JNK inhibitor SP600125 on RCC chemotherapy. RCC cells were cultured with or without IL-33 (50 ng/mL) for 48 hours and SP600125 (10 $\mu \mathrm{g} / \mathrm{mL})$ was added to the culture, and then RCC cells were subsequently exposed to 5-Fu for 24 hours. The cell viability was determined by flow cytometry. Results are expressed as the mean \pm SEM. ${ }^{* *} \mathrm{P}<0.01$. 


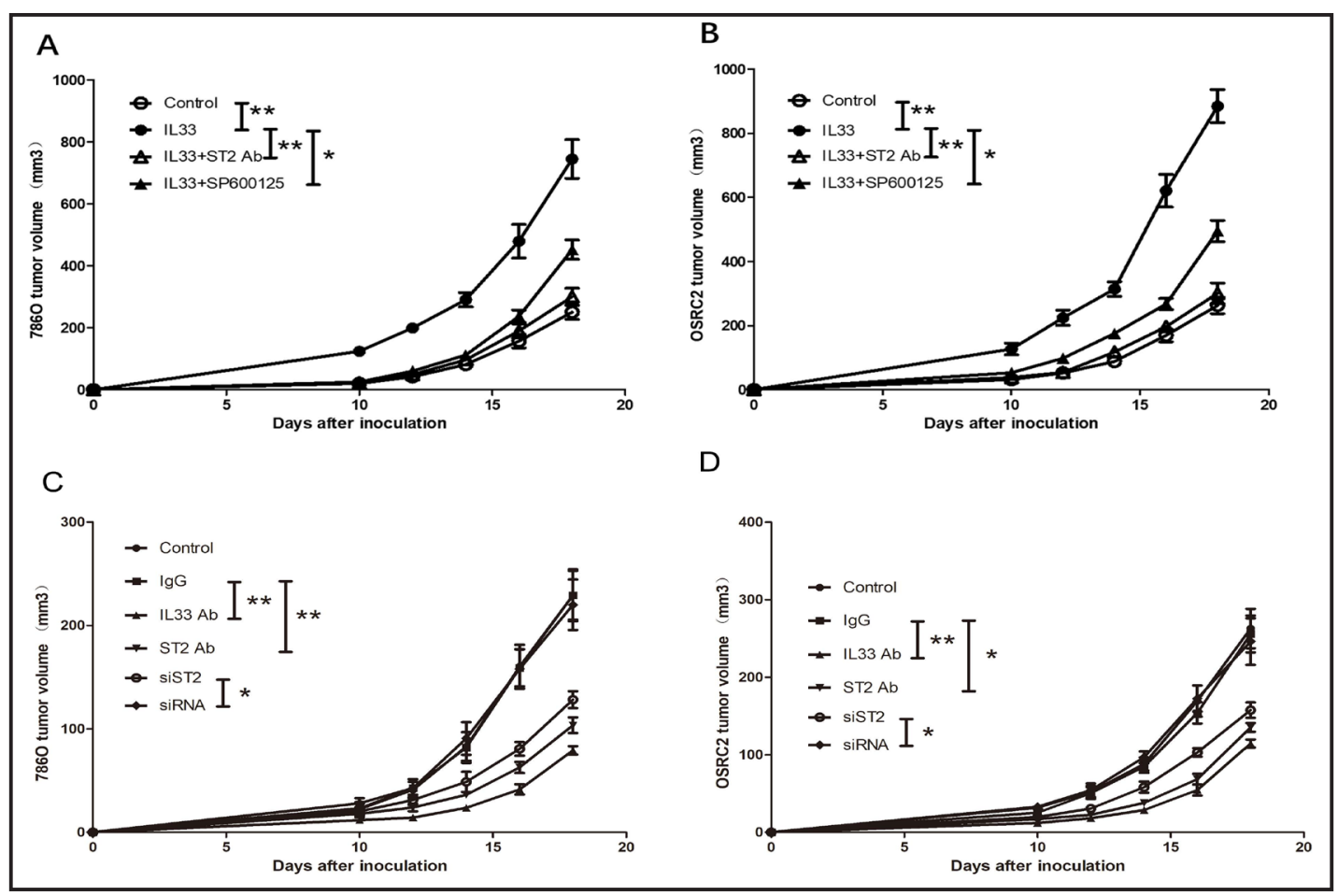

Fig. 5. IL-33 promotes RCC cancer growth via its receptor ST2 and JNK activation in vivo(A-B) OSRC2 and 7860 cells were cultured with or without IL-33 (50 ng/mL) in the presence or absence of anti-ST2 antibody $(1 \mu \mathrm{g} / \mathrm{mL})$ or SP600125 (10 $\mathrm{gg} / \mathrm{mL})$ for 48 hours. The cells (106) were subcutaneously injected into nude mice ( $n=4$ mice/group).. The tumor volume was monitored. Results are expressed as the mean of tumor volume \pm SEM. ${ }^{*} \mathrm{P}<0.05 ;{ }^{* *} \mathrm{P}<0.01$. (C-D) OSRC2 and 7860 cells were transfected with ST2-siRNA (siST2). The cells (106) were subcutaneously injected into nude mice ( $n=4$ mice/group). Neutralizing antibody against either IL-33 or ST2 was injected every two days after RCC cell injection. The tumor volume was monitored. Results are expressed as the mean of tumor volume \pm SEM. ${ }^{*} \mathrm{P}<0.05 ;{ }^{*} \mathrm{P}<0.01$.

IL-33 has been reported to mediate Th2 immune responses ${ }^{6,9,10}$, regulatory T cell (Treg) development in intestinal tissue ${ }^{11}$, and viral-specific CD8+ $\mathrm{T}$ cell functions 12 in murine models. However, the role of IL-33 and the underlying molecular mechanisms in RCC are poorly understood. We demonstrate that IL-33 directly targets and stimulates RCC cell proliferation and prevents 5-Fu-induced tumor cell apoptosis via JNK activation. High levels of IL-33 are expressed in the RCC microenvironment. The protumor role of IL-33 is validated in animal models with IL-33 treatment, and blockade of the IL-33/ST2 signaling pathway abolishes the protumor activities of IL-33. Thus, we suggest that the IL-33/ST2 signaling pathway may be functionally operative in the cancer microenvironment of RCC patients. Indeed, IL-33 administration promotes tumorigenesis ${ }^{14}$, and soluble ST2 is correlated with tumor burden 20, 23. IL-33 predicts poor prognosis and promotes ovarian cancer growth and metastasis ${ }^{24}$ and activates tumor stroma to promote intestinal polyposis 25 . Our results have demonstrated that expression of tumor IL-33 is associated with poor survival in RCC patients. Thus, we reason that the IL-33/ST2 signaling pathway plays a direct protumor role in patients with cancer.

IL-33 binds to ST2 and activates the downstream signaling pathways through NF-kB as well as MAPKs pathways [20], key signaling pathways for cancer cell proliferation. P38 signaling pathway was associated with the pro-melanogenic activity of IL-33 in primary melanocytes [21], while IL-33-promoted proliferation and invasiveness of decidual stromal cells were mediated by both NF-kB and ERK1/2 signaling [10]. To understand the molecular mechanisms underlying the function of IL-33 in RCC cell proliferation and chemotherapy resistance, we examined the phosphorylation of multiple MAPKs, including ERK1/2, JNK, 


\section{Cellular Physiology Cell Physiol Biochem 2018;47:191-200

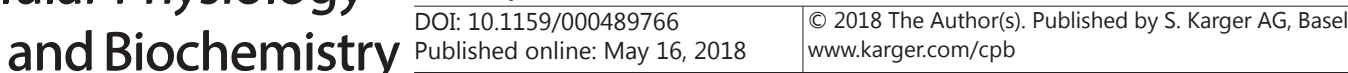 \\ Wu et al.: IL-33 Promotes RCC Cell Proliferation}

and P38 in the RCC cell lines. Our results identified important roles for the JNK signaling pathways in IL-33-mediated aggressive behavior of RCC cells as there was a dramatic increase in the phosphorylation of JNK after IL-33 stimulation, and a JNK-specific inhibitor, SP600125, abolished the effects of IL-33 on RCC cell proliferation and chemotherapy resistance in vivo and in vitro. However, neither ERK or P38 phosphorylation was detected, suggesting they were not involved in IL-33-mediated RCC cell proliferation and chemotherapy resistance.

In conclusion, we demonstrate that that increased expression of IL-33 in RCC was associated with advanced TNM stage and inversely correlated with prognosis. IL-33 enhances RCC cell growth in vivo and stimulates RCC cell proliferation and prevents chemotherapyinduced tumor apoptosis in vitro. Furthermore, we have demonstrated that IL-33 promotes RCC cell proliferation and chemotherapy resistance via its receptor ST2 and the JNK signaling activation in tumor cells. Our work suggests that targeting IL-33/ST2 and JNK signaling may be potentially applicable in treating patients with RCC.

\section{Acknowledgements}

We sincerely thank "Editage" to offer professional English language editing to us, and Natural Science Foundation of JiangXi province (20132BAB205010) to fund this research.

\section{Disclosure Statement}

The authors declare that there is no conflict of interest.

\section{References}

1 Torre LA, Bray F, Siegel RL, Ferlay J, Lortet-Tieulent J, Jemal A: Global cancer statistics, 2012. CA Cancer J Clin 2015;65:87-108.

-2 Ljungberg B, Bensalah K, Canfield S, Dabestani S, Hofmann F, Hora M, Kuczyk MA, Lam T, Marconi L, Merseburger AS, Mulders P, Powles T, Staehler M, Volpe A, Bex A: EAU guidelines on renal cell carcinoma: 2014 update. Eur Urol 2015;67:913-924.

-3 Pirrotta MT, Bernardeschi P, Fiorentini G: Targeted-therapy in advanced renal cell carcinoma. Curr Med Chem 2011;18:1651-1657.

4 Chen W, Hill H, Christie A, Kim MS, Holloman E, Pavia-Jimenez A, Homayoun F, Ma Y, Patel N, Yell P, Hao G, Yousuf Q, Joyce A, Pedrosa I, Geiger H, Zhang H, Chang J, Gardner KH, Bruick RK, Reeves C, Hwang TH, Courtney K, Frenkel E, Sun X, Zojwalla N, Wong T, Rizzi JP, Wallace EM, Josey JA, Xie Y, Xie XJ, Kapur P, McKay RM, Brugarolas J: Targeting renal cell carcinoma with a HIF-2 antagonist. Nature 2016;539:112-117.

5 Dinarello CA: An IL-1 family member requires caspase-1 processing and signals through the ST2 receptor. Immunity 2005;23:461-462.

-6 Schmitz J, Owyang A, Oldham E, Song Y, Murphy E, McClanahan TK, Zurawski G, Moshrefi M, Qin J, Li X, Gorman DM, Bazan JF, Kastelein RA: IL-33, an interleukin-1-like cytokine that signals via the IL-1 receptorrelated protein ST2 and induces T helper type 2-associated cytokines. Immunity 2005;23:479-490.

7 Villarreal DO, Wise MC, Walters JN, Reuschel EL, Choi MJ, Obeng-Adjei N, Yan J, Morrow MP, Weiner DB: Alarmin IL-33 acts as an immunoadjuvant to enhance antigen-specific tumor immunity. Cancer Res 2014;74:1789-1800.

8 Liew FY, Pitman NI, McInnes IB: Disease-associated functions of IL-33: the new kid in the IL-1 family. Nat Rev Immunol 2010;10:103-110.

-9 Maywald RL, Doerner SK, Pastorelli L, De Salvo C, Benton SM, Dawson EP, Lanza DG, Berger NA, Markowitz SD, Lenz HJ, Nadeau JH, Pizarro TT, Heaney JD: IL-33 activates tumor stroma to promote intestinal polyposis. Proc Natl Acad Sci U S A 2015;112:E2487-2496. 


\section{Cellular Physiology Cell Physiol Biochem 2018;47:191-200 \begin{tabular}{l|l} 
DOI: 10.1159/000489766 & Ond 2018 The Author(s). Published by S. Karger AG, Basel \\
www.karger.com/cpb
\end{tabular} \\ Wu et al.: IL-33 Promotes RCC Cell Proliferation}

10 Hu WT, Li MQ Liu W, Jin LP, Li DJ, Zhu XY: IL-33 enhances proliferation and invasiveness of decidual stromal cells by up-regulation of CCL2/CCR2 via NF-kappaB and ERK1/2 signaling. Mol Hum Reprod 2014;20:358-372.

11 Yamada D, Rizvi S, Razumilava N, Bronk SF, Davila JI, Champion MD, Borad MJ, Bezerra JA, Chen X, Gores GJ: IL-33 facilitates oncogene-induced cholangiocarcinoma in mice by an interleukin-6-sensitive mechanism. Hepatology 2015;61:1627-1642.

-12 Kurowska-Stolarska M, Kewin P, Murphy G, Russo RC, Stolarski B, Garcia CC, Komai-Koma M, Pitman N, Li Y, Niedbala W, McKenzie AN, Teixeira MM, Liew FY, Xu D: IL-33 induces antigen-specific IL-5+ T cells and promotes allergic-induced airway inflammation independent of IL-4. J Immunol 2008;181:4780-4790.

-13 Jones LA, Roberts F, Nickdel MB, Brombacher F, McKenzie AN, Henriquez FL, Alexander J, Roberts CW: IL33 receptor (T1/ST2) signalling is necessary to prevent the development of encephalitis in mice infected with Toxoplasma gondii. Eur J Immunol 2010;40:426-436.

14 Tordesillas L, Goswami R, Benede S, Grishina G, Dunkin D, Jarvinen KM, Maleki SJ, Sampson HA, Berin MC: Skin exposure promotes a Th2-dependent sensitization to peanut allergens. J Clin Invest 2014;124:49654975.

15 Li J, Razumilava N, Gores GJ, Walters S, Mizuochi T, Mourya R, Bessho K, Wang YH, Glaser SS, Shivakumar P, Bezerra JA: Biliary repair and carcinogenesis are mediated by IL-33-dependent cholangiocyte proliferation. J Clin Invest 2014;124:3241-3251.

16 Gao X, Wang X, Yang Q, Zhao X, Wen W, Li G, Lu J, Qin W, Qi Y, Xie F, Jiang J, Wu C, Zhang X, Chen X, Turnquist H, Zhu Y, Lu B: Tumoral expression of IL-33 inhibits tumor growth and modifies the tumor microenvironment through CD8+ T and NK cells. J Immunol 2015;194:438-445.

17 Mertz KD, Mager LF, Wasmer MH, Thiesler T, Koelzer VH, Ruzzante G, Joller S, Murdoch JR, Brummendorf T, Genitsch V, Lugli A, Cathomas G, Moch H, Weber A, Zlobec I, Junt T, Krebs P: The IL-33/ST2 pathway contributes to intestinal tumorigenesis in humans and mice. Oncoimmunology 2016;5:e1062966.

18 Akimoto M, Hayashi JI, Nakae S, Saito H, Takenaga K: Interleukin-33 enhances programmed oncosis of ST2L-positive low-metastatic cells in the tumour microenvironment of lung cancer. Cell Death Dis 2016; 7:e2057.

19 Jovanovic IP, Pejnovic NN, Radosavljevic GD, Pantic JM, Milovanovic MZ, Arsenijevic NN, Lukic ML: Interleukin-33/ST2 axis promotes breast cancer growth and metastases by facilitating intratumoral accumulation of immunosuppressive and innate lymphoid cells. Int J Cancer 2014;134:1669-1682.

20 Liew FY: IL-33: a Janus cytokine. Ann Rheum Dis 2012;71 Suppl 2:1101-104.

21 Zhou J, Song J, Ping F, Shang J: Enhancement of the p38 MAPK and PKA signaling pathways is associated with the pro-melanogenic activity of Interleukin 33 in primary melanocytes. J Dermatol Sci 2014;73:110116. 